\title{
Shape selectivity vapor-phase conversion of lignin-derived 4-ethylphenol to phenol and ethylene over acidic aluminosilicates: Impact of acid properties and pore constraint
}

\author{
Yuhe Liao, Martin d'Halluin, Ekaterina Makshina, Danny Verboekend*, Bert F. Sels* \\ Center for Surface Chemistry and Catalysis, Katholieke Universiteit Leuven, Celestijnenlaan 200F, 3001, Heverlee, Belgium
}

\section{A R T I C L E I N F O}

\section{Keywords:}

Dealkylation

Shape selectivity

Aluminosilicate

ZSM-5

Ethylphenol

Bio-Phenol

\begin{abstract}
A B S T R A C T
Selective dealkylation of alkylphenols, the opposite reaction of the more commonly studied phenol alkylation, may represent an important reaction in the production of base chemicals like phenol and olefins from fossilized and raw lignocellulosic matter. This study reports the first thermodynamics and kinetics studies of the vaporphase conversion of ethylphenol (EP) over acidic $\gamma$-Al2O3, amorphous (ASA) and crystalline aluminosilicates (like ferrierite, ZSM-22, ZSM-5, beta, and USY) in the absence of hydrogen and noble metals, as a way to produce phenol and ethylene. The reaction was studied deliberately in presence of steam to get stable time on stream catalysis. The thermodynamic analysis shows an endothermal EP conversion to phenol and ethylene, favoured at high reaction temperature, while isomerisation, disproportionation and transalkylation are thermodynamically preferred at low temperature. The kinetic study examines the role of the catalytically active sites; it reveals the importance of site constraint on the activity, selectivity and stability, and shows the complex temperature dependency of the dealkylation. Both Brønsted and Lewis acid sites are active, but multifactor dependency (such as acid strength and site accessibility) complicates the establishment of simple quantitative relationships with the acid type. EP does not enter the micropores of ferrierite and ZSM-22, as suggested by adsorption experiments. Kinetics without significant diffusion limitations were obtained with ZSM-5, beta and USY. Thus, in absence of intracrystalline diffusion limitation (as verified by calculations using reported effective diffusivities, and substantiated by a comparably high apparent activation energy for all zeolites), the increased reaction turnover rate with increasing pore size from medium to large pore zeolites is largely explained by a change in reaction pathway (from monolecular to bimolecular) to convert EP to phenol and ethylene. A pathway proceeding through fast thermodynamically favourable bimolecular reactions occurs in the spatially non-constrained pores and crystal surface, whereas monomolecular reactions take place in the micropores of ZSM-5. Despite the lower rate, the selectivity over ZSM-5 strongly benefits from active site confinement, being responsible to achieve quantitative formation of phenol and ethylene from ethylphenol. The excellent performance of ZSM-5 thus accords with its shape selective property that avoids undesired side reactions such as the sterically demanding bimolecular reactions like disproportionation, transalkylation and C-C cracking, and severe cokes formation.
\end{abstract}

\section{Introduction}

The extensive worldwide utilization of fossil oil may lead to a longterm shortage of bulk chemicals. A partial potential solution to replace and/or compensate the oil-based chemicals is the identification of a secondary source of base chemicals. Aromatics are one of the major platform compounds for today's chemical industry. As raw and fossilized lignocelluloses contain aromatic structures, they may offer potential for the production of aromatic compounds [1-5]. The aromatic chemicals obtained from lignocellulose are usually alkylated aromatics
[3,6-20]. Among others, ethyl- (EPs) and propylphenols (PPs) are common aromatics derived from lignin depolymerization and the gasification of coal [3,6,21-26]. Alkylphenols have interesting application themselves like solvents, fuel and rubber additives, and thermosets [27-31]. Valorization of these alkylphenols into base chemicals like phenol and olefins is also relevant when they become abundantly available, but this synthetic pathway is underexplored.

Cleavage of the alkyl group in alkylphenols can be achieved by using several non-catalytic and catalytic technologies (Table S1). Thermal (hydro-) cracking is one of the most important non-catalytic

\footnotetext{
* Corresponding authors.

E-mail addresses: danny.verboekend@kuleuven.be (D. Verboekend), bert.sels@kuleuven.be (B.F. Sels).
} 
one, which yields phenol as the main product. However, such thermolytic reaction produces a lot of side products (ca. 40\%), because of the high process temperature $(>773 \mathrm{~K}$ ) to obtain conversions exceeding 30\% [32-34]. Catalytic hydrocracking can occur at milder temperature with a selectivity of $70 \%$. Still, products from the alkyl side chain are alkanes, whereas formation of alkenes is more desirable [35]. Besides exploration of mature technologies, dealkylation was also studied in supercritical water, yielding a very low phenol selectivity (42\%), having methane and $\mathrm{CO}_{2}$ as main side products [36]. Catalytic steam dealkylation was developed to convert cresols over noble metal with $70 \%$ selectivity to phenol [37]. Despite these successful alkyl chain removals, the selectivity to the most valuable chemicals, viz. phenol and olefin, is moderate $(<70 \%)$. Besides, hydrocracking and steam dealkylation, consume costly hydrogen and/or require an additional metal function. Furthermore, the typical catalysts of the catalytic dealkylation process deactivate rapidly. There is thus clearly a need to explore more attractive alternatives that combine a lower process temperature, preferably below $773 \mathrm{~K}$, with a high selectivity to phenol and olefin, while avoiding the use of hydrogen and costly noble metals.

The available technologies of ethylbenzene dealkylation might inspire solutions for converting EPs. The reactivity of EPs is expected to be substantially higher than that of ethylbenzene over the same acidic catalyst. The presence of the phenolic group is likely beneficial for substrate adsorption and formation of the activated complex [38]. It also adds on reaction complexity due to the potential formation of positional isomers.

Dealkylation of EPs to phenol has been reported over an acidic fluoborate catalyst and a silica-alumina catalyst [38,39]. Catalytic conversion of alkylphenols in gas phase to phenol and alkenes was recently proposed over acidic zeolites [4], representing an outstanding opportunity to upgrade depolymerized lignin streams with the use of zeolites [40-42]. Therein, it was shown that the combination of a zeolite and steam is essential to obtain a stable catalytic performance. This zeolite-catalysed reaction with alkylphenols is fundamentally different from the classic ethylbenzene dealkylation transformation, for which $\mathrm{H}_{2}$ and Pt are required to achieve stable catalysis, while lowvalue ethane is formed as by-product [35]. Yet, several aspects clearly deserve more attention, such as the exact nature of the active sites, the effect of acid strength and density, pore constraints, definition of primary and secondary reaction types and their relationship in a reaction network, thermodynamic vs. kinetic aspects, and mass transfer issues. In addition, the critical role of the zeolite porosity (e.g., on the uptake of alkylphenols), and the effect of pore structure on the activity, stability and selectivity have not been investigated.

Herein, we present a thorough investigation of the catalytic dealkylation of EP into phenol and ethylene over acidic aluminosilicates. The thermodynamic aspects are handled first, followed by a first kinetic exploration of several Lewis and/or Brønsted solid acids, to ultimately unravel the requirements and role of the active sites and to point out the superiority of (some) zeolites. A network of different reaction pathways is proposed based on a study of the product evolution in function of contact time and temperature. The importance of the pore geometry of zeolites (topology), acid strength and number of active sites on activity, selectivity, stability and reaction pathway is demonstrated, evidencing ZSM-5 as the most selective catalyst mainly due to pore constraint. Optimal catalyst and process conditions are formulated to achieve a high phenol and olefin selectivity at high EP conversion, high productivity, and a stable catalytic performance.

\section{Experimental section}

\subsection{Materials}

4-Ethylphenol (4-EP) (98\%), 2-ethylphenol (2-EP) (99\%), and 3ethylphenol (3-EP) (80\%, remainder mainly 4-EP) were supplied by Sigma Aldrich, and used as the reaction substrates without any further treatment. All the commercial catalysts employed and the corresponding codes in this contribution are listed in Table S2. These catalysts were calcined in static air at $823 \mathrm{~K}$ for $5.5 \mathrm{~h}$ with a ramping rate $5 \mathrm{~K} \mathrm{~min}^{-1}$. All the zeolite catalysts were transferred into protonic form before the catalytic evaluation. beta-P was prepared by post modification of beta-19 according to the reported methods [43].

\subsection{Catalysts characterization}

Nitrogen-sorption ( $\mathrm{N}_{2}$-sorption) measurements were conducted at $77 \mathrm{~K}$ with a Micrometrics Tristar 3000 instrument. Prior to the measurement, the samples are degassed at $573 \mathrm{~K}$ under a flow of $\mathrm{N}_{2}$ for overnight. The BET method was applied to calculate the surface area. The Barret-Joyner-Halenda (BJH) model was applied to the adsorption branch of isotherm for obtaining the pore size distribution. The total volume was the volume adsorbed at $\mathrm{p} / \mathrm{p}_{0}=0.99$. The $\mathrm{t}$-plot method was applied to calculate the micropore volume. The texture properties of all samples are summarized in Table S3. The $\mathrm{N}_{2}$ isotherms and associated pore size distributions are presented in Figs. S1 and S2. The carbon content of spent zeolites was analyzed by elemental analyzer (vario EL cube, Elementar). Pyridine FTIR measurements were conducted in a Nicolet 6700 spectrometer, equipped with a DTGS detector (256 scans per spectrum, $2 \mathrm{~cm}^{-1}$ resolution). Self-supporting sample wafers were degassed at $673 \mathrm{~K}$ for $1 \mathrm{~h}$ under vacuum before measurements. A pyridine probe was employed to analyze Brønsted (B) and Lewis (L) acid sites. Afterwards, the samples were subjected to $25 \mathrm{mbar}$ of pyridine vapor at $323 \mathrm{~K}$ until saturation. Then, the sample was degassed at $423 \mathrm{~K}, 523 \mathrm{~K}$, and $623 \mathrm{~K}$ before measuring at $423 \mathrm{~K}$. The band areas of adsorbed pyridine at 1550 and $1450 \mathrm{~cm}^{-1}$ corresponded to the total concentration of Brønsted $\left(C_{B}\right)$ and Lewis $\left(C_{L}\right)$ acid sites, respec-

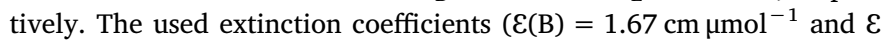

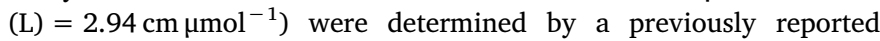
method [44]. The results of pyridine FTIR measurements are listed in Table S3. Thermo-gravimetric analysis (TGA) was conducted by using a TGA-Q500 analyzer. The measurements were conducted in air $\left(30 \mathrm{~cm}^{3} \mathrm{STP} \mathrm{min}^{-1}\right)$ ramping the temperature from 303 to $1073 \mathrm{~K}$ at $10 \mathrm{~K} \mathrm{~min}^{-1}$. Ammonia temperature-programmed desorption $\left(\mathrm{NH}_{3}-\right.$ TPD) measurements were conducted in a flow apparatus equipped with a Pfeiffer Omnistar quardrupole mass spectrometer for the desorbed gas $\left(\mathrm{NH}_{3}\right)$ [45]. For this, the sample $(100 \mathrm{mg})$ was first pretreated in a helium flow at $673 \mathrm{~K}$ (ramping rate: $5 \mathrm{~K} \mathrm{~min}^{-1}$ ) for $2 \mathrm{~h}$. The adsorption of $\mathrm{NH}_{3}$ was performed at $473 \mathrm{~K}$ for $0.5 \mathrm{~h}$, followed by flushing with helium for $0.5 \mathrm{~h}$ at the same temperature. The sample was heated to $1273 \mathrm{~K}$ with a ramping rate of $10 \mathrm{~K} \mathrm{~min}^{-1}$ in a helium flow. $\gamma-\mathrm{Al}_{2} \mathrm{O}_{3}$ was used as a reference material to quantify the acidity. The $\mathrm{NH}_{3}$-TPD profiles are presented in Figs. S1 and S3.

\subsection{Catalytic evaluation}

The vapor-phase dealkylation experiments were performed in a custom-built fixed-bed reactor equipped with four parallel quartz reactor tubes (i.d. of $3 \mathrm{~mm}$ ) under ambient pressure. Typically, the powder catalyst was tableted and pulverized into $0.125-0.25 \mathrm{~mm}$ before catalytic test. In a typical procedure, a $120 \mathrm{mg}$ catalyst was loaded into the four quartz reactor tubes ( $30 \mathrm{mg}$ per tube) and held by two layers of quartz wool, generating a catalyst bed height of $c a .13 \mathrm{~mm}$. EPs and water were fed into the gas phase by flowing nitrogen gas through a heated saturator. Subsequently, a gas mixture of molar composition: 0.86/0.12/0.02 $\left(\mathrm{N}_{2} / \mathrm{H}_{2} \mathrm{O} / \mathrm{EP}\right)$ was obtained, and introduced into the reactors. Water was fed in the reaction stream to sufficiently stabilize the catalytic activity (Fig. S4). The catalytic performance as function of temperature was conducted with a heating rate of $1 \mathrm{~K} \mathrm{~min}^{-1}$. In order to understand the impact of hysteresis effects on the temperature dependency (e.g., due to catalyst destabilization), control experiments at single temperature (at steady state) and reverse light-off experiments (with a drop rate of $1 \mathrm{~K} \mathrm{~min}^{-1}$ ), subsequent to the 
normal light-off experiment were conducted (Fig. S5). The outcome of the control experiments show sufficient reliability of the light-off curves as a practical way to investigate the temperature dependency of EP conversion over the catalysts. The weight hourly space velocity (WHSV) experiments were conducted by reducing the amount of catalyst, while keeping the height of catalyst bed by diluting the catalyst with quartz powder $(0.125-0.25 \mathrm{~mm})$. For the kinetic study, the conversion is usually lower than $5 \%$, and sometimes below $10 \%$, as to be able to calculate the apparent activation energies from the differential reaction rates. The external and internal mass diffusion were evaluated by changing the particle size and by changing the flow rates at the same space velocity, respectively (Fig. S6). Meanwhile, Mears and WeiszPrater criteria were also used to discuss external and internal mass diffusion issues, respectively (see Supporting information) [46,47]. Effective diffusion coefficient $D_{e}$ of 4-EP was estimated based on the reported $D_{e}$ of similar aromatics and their trends (Table S4), and assumes $D_{e}$ of 4-EP larger than $10^{-15} \mathrm{~m}^{2} \mathrm{~s}^{-1}$. All these classic control experiments and calculations indicate absence of diffusion limitations for ZSM-5, beta and USY.

The products line was heated to $473 \mathrm{~K}$ to avoid condensation of high boiling point products. The products from the reactor outlet were monitored using an on-line Gas Chromatography (GC HP4890D) equipped with Flame Ionization Detector (FID) and a HP1 column. High boiling point $(>313 \mathrm{~K}$ ) products were collected by a collector with methanol at $303 \mathrm{~K}$. The collected products were analyzed using an Agilent Technologies 7890A Gas Chromatograph equipped with a FID and mass spectrometer and a HP1 column. The results of GC-MS were compared with the results of online GC to guarantee that the online GC can monitor all products.

The thermodynamic equilibrium compositions of EP were calculated by using the Gibbs reactor of ASPEN PLUS (V8.6). In this work, the online GC cannot distinguish 4-EP and 3-EP. EPs involves 4-EP, 3-EP and 2-EP. The presence of 3-EP in the products was determined using off-line GC. Cresols includes ortho-, meta-, and para-cresol. The ratio of water/4-EP refers to the molar ratio.

\subsection{Liquid-phase adsorption}

Adsorption isotherms were obtained by immersing $0.5 \mathrm{~g}$ of zeolites in a given concentration of 4-EP in toluene $(10 \mathrm{~mL})$ for $12 \mathrm{~h}$. The liquid was separated from the zeolite by centrifugation and the residual concentration of 4-EP in the toluene was determined by GC using internal standard ( $m$-xylene). The uptake was determined by the change in the bulk concentration.

\section{Results and discussion}

\subsection{Thermodynamic considerations of the reaction under study}

4-EP was selected as a model compound for this dealkylation study since the ethyl group of lignin-derived EPs is typically positioned para to the hydroxyl group [6,21-23]. Reported results of the analogue ethylbenzene dealkylation demonstrated the occurrence of disproportionation in addition to dealkylation [48-52]. Some anticipated key reactions (R1-R8) are listed in Fig. 1. Dealkylation (R1 and R3, Fig. 1) is cleavage of the $\mathrm{C}-\mathrm{C}$ bond between the aromatic ring and ethyl group, which produces phenol and olefin. Due to the presence of the phenolic group, positional isomerisation may occur (R7, Fig. 1) [39]. Disproportionation (R5, Fig. 1) is the transfer of the ethyl group from one 4-EP molecule to another 4-EP molecule, whereas transalkylation is the transfer of the ethyl group from one 4-EP molecule to a different molecule (like other isomers of EP or phenol), which is also a possible for 4-EP. The thermodynamics of transalkylation were not shown here, as it is very similar to that of disproportionation. Besides the above reactions, 4-EP may be subjected to terminal C-C cracking in the ethyl group, especially at high temperature, which gives rise to cresol. The thermodynamic aspect of this reaction was not shown, as it is a minor reaction of 4-EP.

Variation of the free Gibbs energy for dealkylation of 4-EP is plotted against temperature in Fig. 1a. In agreement with the energy requirement for cleavage of a $\mathrm{C}-\mathrm{C}$ bond, dealkylation (R1 and R3, Fig. 1) is an endothermic reaction (with $\Delta \mathrm{H}=103.6 \mathrm{~kJ} \mathrm{~mol}^{-1}$ ), and therefore favourable at high temperature. The opposite (multi-)alkylation of phenol with ethylene to EP (R2, Fig. 1) and diEPs (R4, Fig. 1) is favourable at low temperature. The variation of the free Gibbs energy $(\Delta G)$ of disproportionation (R5, Fig. 1) and isomerisation (R7, Fig. 1) of 4-EP is also plotted as a function of temperature in Fig. 1a. As the free Gibbs energies are almost constant (close to zero) over the entire temperature range, viz $473-773 \mathrm{~K}$, and that of dealkylation is positive at low temperature, the two reactions (isomerisation and disproportionation) are particularly dominant in the low temperature range. The thermodynamic analysis thus clearly illustrates the necessity of a sufficiently high process temperature to achieve selective (and complete) dealkylation of 4-EP. Fig. 1b illustrates the product distribution of phenol alkylation with ethylene (with phenol to ethylene molar ratio of 1). EP isomers rather than phenol are the dominant products at low temperature in line with the above analysis. The molar ratio of 3- and 4-EP to 2 -EP equals 2.5 , the equilibrium value being largely temperature independent (Fig. 1c).

\subsection{Exploration of different commercially available catalyst types}

Cleavage of aromatic ring-alkyl $\mathrm{C}-\mathrm{C}$ bond is generally associated with acid catalysis, but several reports have also demonstrated dealkylation over basic oxides like $\mathrm{ZnO}$ and $\mathrm{MgO}[54,55]$. Therefore, a series of commercial oxides with different active site types were initially explored for 4-EP dealkylation (Fig. 2a). Commercial $\mathrm{ZnO}, \mathrm{ZrO}_{2}$, and $\mathrm{MgO}$ catalysts show very low conversion rates, likely because of their low surface area (in case of $\mathrm{ZnO}$ and $\mathrm{ZrO}_{2}$ ) and/or limited/absent acidity (in case of all three) (Fig. S1). These oxides were not further discussed. Since aluminium fluoborate (5 wt.\%) deposited on $\gamma-\mathrm{Al}_{2} \mathrm{O}_{3}$ can convert 4-EP [39], $\gamma-\mathrm{Al}_{2} \mathrm{O}_{3}$ was investigated and used as a reference material to investigate other acidic catalysts.

The high conversion rates of both the amorphous silica alumina (ASA, with $185 \mu \mathrm{mol} \mathrm{g}^{-1}$ Lewis and $21 \mu \mathrm{mol} \mathrm{g}^{-1}$ Brønsted acidity, respectively. Table S3) and $\gamma-\mathrm{Al}_{2} \mathrm{O}_{3}$ (with 103 and $0 \mu \mathrm{mol} \mathrm{g}^{-1}$ Lewis and Brønsted acidity, respectively. Table S3) suggest an essential role of acid sites. The activity of $\gamma-\mathrm{Al}_{2} \mathrm{O}_{3}$ directly indicates that Lewis acid sites are active in the dealkylation of EPs. Zeolite ZSM-5-P with 40 and $177 \mu \mathrm{mol} \mathrm{g}^{-1}$ Lewis and Brønsted acidity, respectively, was selected to evaluate the role of Brønsted acid sites. The substantial conversion rate of ZSM-5-P suggests that also Brønsted acid sites have an important role to play in the reaction.

The catalytic activity of acid catalysts is corroborated by testing different acidic (amorphous and crystalline) aluminosilicates. Their activities, expressed per mol acid sites, and temperature dependency were compared in the kinetic regime (see experimental section and supporting information). The Arrhenius plots are shown in Fig. 2b. The results of ZSM-5-P and $\gamma-\mathrm{Al}_{2} \mathrm{O}_{3}$ confirm that both Lewis and Brønsted sites are very active in the dealkylation of EPs. Despite the knowledge of the individual activity per acid site type, we fail to predict the precise activity of catalysts with mixed acid site types (see Supporting information. Fig. S7). This suggests that not only the acid type, but more complex relationships with regard to the acid strength, accessibility etc. govern the activity. Though different contributions may thus be expected from both acid site types, TOFs in this contribution are further calculated based on the total acidity (based on Pyridine FTIR) for simplicity. Especially in case of zeolites, which are the core of this work and contain mainly Brønsted acid sites, such simplification of TOF calculations is defendable.

Accordingly, USY is the most active catalyst, while ZSM-22-P and FER-P show very low activity (Fig. 2b). Despite the activity difference, 

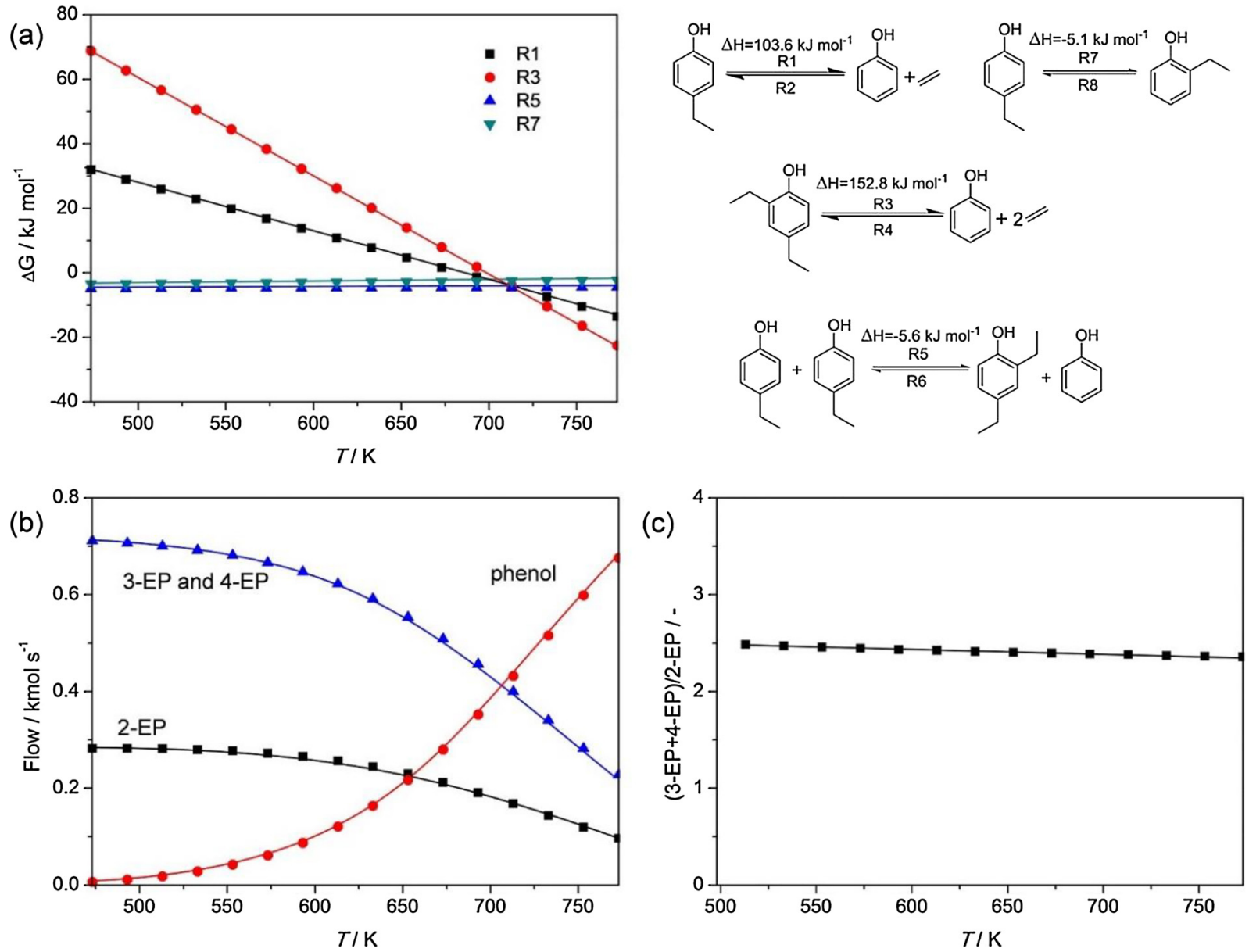

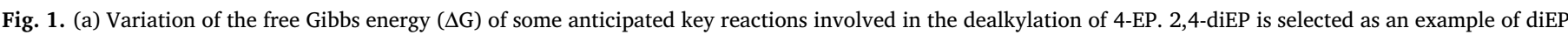

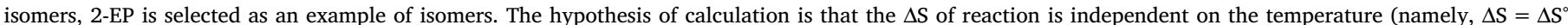

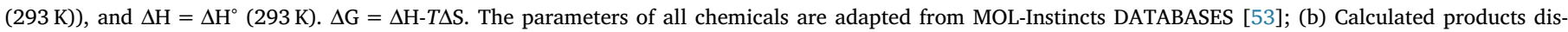

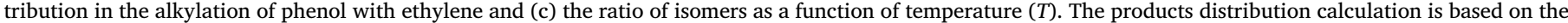

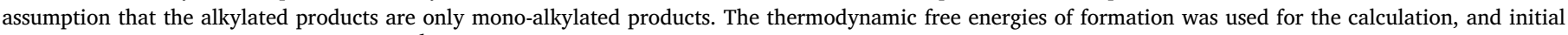
flow of phenol and ethylene was $1 \mathrm{kmol} \mathrm{s}^{-1}$ in the calculation. EP is ethylphenol, diEPs is diethylphenols.

all zeolites (irrespective of topology and $\mathrm{Si}$ to $\mathrm{Al}$ ratio) show very similar apparent activation energy values, around $60 \mathrm{~kJ} \mathrm{~mol}^{-1}$ (Fig. 2b), suggesting a similar reaction mechanism (as they are rich in Brønsted acid sites with comparably low Lewis acidity; Table S3). Hence, the different activities are due to differences in pre-exponential factor, and therefore linked to entropic factors, including site accessibility and catalytic site effectivity of reaction.

As the zeolites used in this works have similar acid strength (except for FER-P, see Fig. S3), the increase of activity (TOF, thus normalized to per mol active sites) with pore size, as suggested by the plot in Fig. 3a,
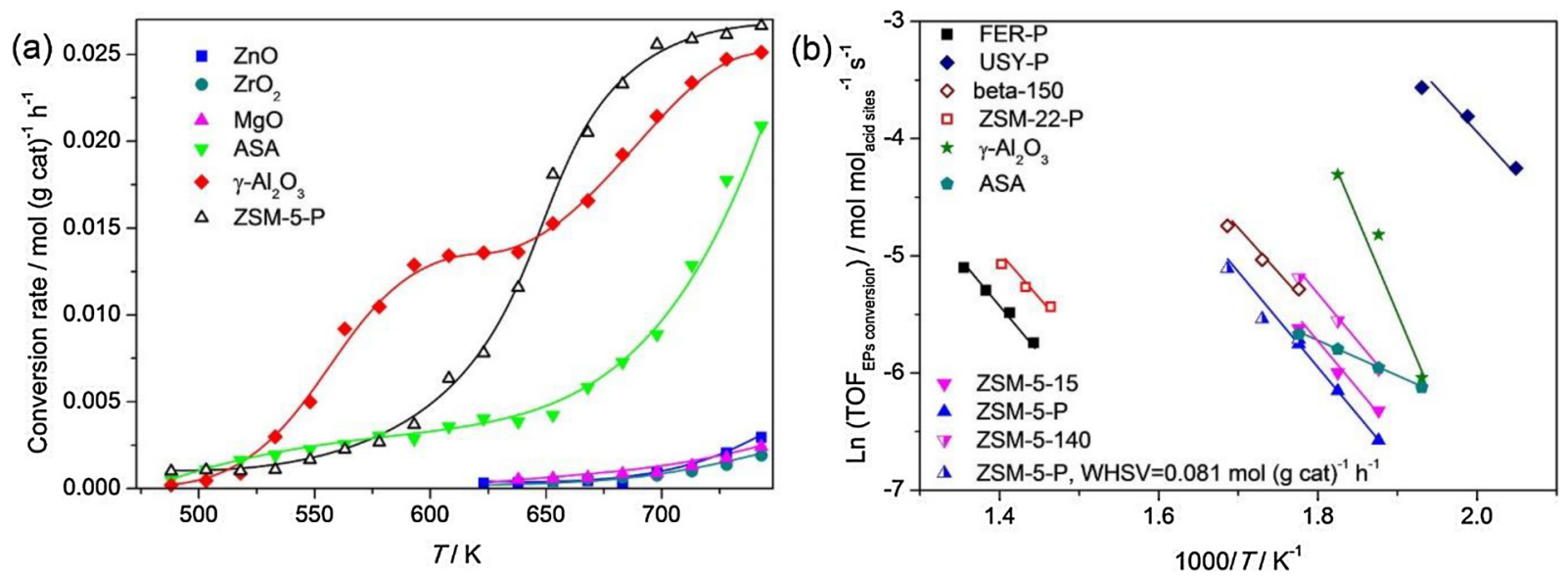

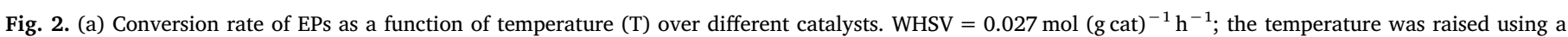

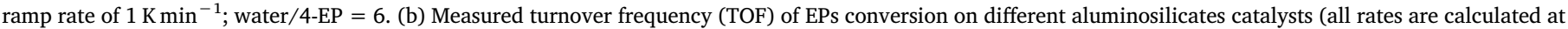
low conversion $<10 \%)$. The acidity was measured by pyridine FTIR. Except when indicated, otherwise WHSV $=0.027 \mathrm{~mol}(\mathrm{~g} \text { cat })^{-1} \mathrm{~h}^{-1}$. 

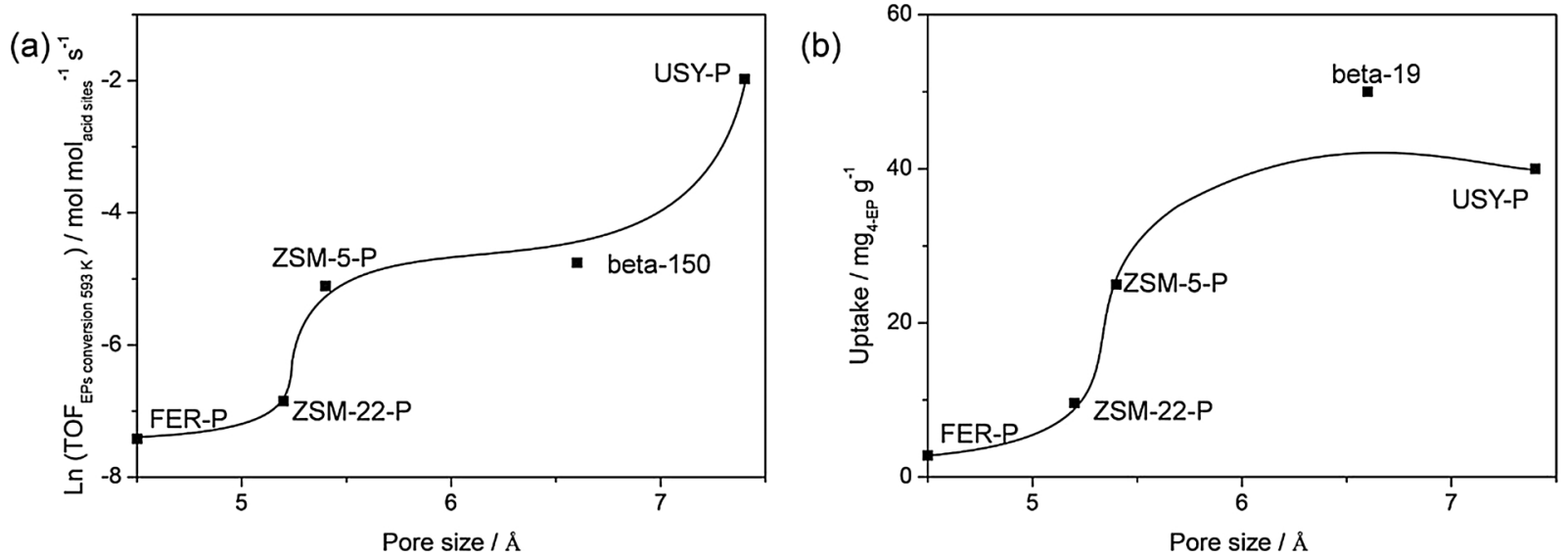

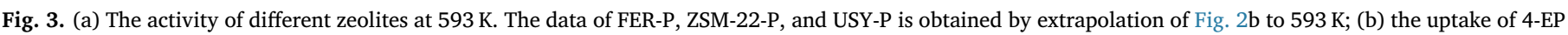
on different zeolites at $313 \mathrm{~K}$.

may be an indication of pore diffusion limitation. Most likely, the activity increase from FER-P over ZSM-22-P to ZSM-5-P is contributed by the enhanced access of EP into the micropore. In case of ZSM-22-P and FER-P, EP can only react on the external surface, as the kinetic diameter of the benzene ring is larger than the size of the micropores. This lack of zeolite crystal entrance is confirmed by adsorption isotherms of 4-EP (from toluene) into zeolites, measured at temperatures between $313 \mathrm{~K}$ to $353 \mathrm{~K}$ (Fig. 3b and S8). The uptake of 4-EP on zeolites follows a Langmuir type adsorption isotherm (Fig. S8). The uptake results, presented in Fig. 3b, indeed demonstrate a restricted access of 4-EP loading in the $4.6 \times 5.7 \AA$ pores of ZSM-22-P and $4.2 \times 5.4 \AA$ and $3.5 \times 4.8 \AA$ pores of FER-P, while a substantial amount of 4-EP is loaded in ZSM-5-P (with pore dimensions of $5.3 \times 5.6 \AA$ and $5.1 \times 5.5 \AA$ ), and even more in beta and USY-P. The low activity of ZSM-22 and FER-P is therefore explained by restricted access to the internal sites throughout the micropores of the zeolite crystal. The adsorption study shows that ZSM-5, beta, and USY are able to adsorb substantial amounts of EP in their micropores, and therefore access of EP in these medium to large pore zeolites is demonstrated.

Assuming an equal accessibility of the active sites in the zeolite series from ZSM-5-P over beta-P to USY-P, the increasing activity for those zeolites can only be explained by a higher conversion efficiency of the active site. Control kinetic experiments (Fig. S6) and calculations (Mears and Weisz-Prater criteria assuming $D_{e}$ of EP is larger than $10^{-15} \mathrm{~m}^{2} \mathrm{~s}^{-1}$; see supporting information) indeed showed absence of film and inter- and intrapore mass transfer issues with EP in the observed reaction rates for these zeolites. Even in absence of pore diffusion, pore size restriction may affect the rate of the reaction by inducing a change in reaction pathway, and as such altering the conversion efficiency of the sites. For instance, one could imagine that by not permitting the faster sterically demanding bimolecular reactions, e.g., disproportionation of 4-EP into diEPs, the conversion over ZSM-5-P will be slower than that over USY. Proof for this hypothesis is elaborated into more detail in the next section.

\subsection{Effect of pore structure of zeolites}

In zeolite catalysis, the pore structure may influence not only the activity but also the reaction selectivity, and the catalyst stability. Therefore, the effect of pore structure was comprehensively studied over the different zeolites. $\gamma-\mathrm{Al}_{2} \mathrm{O}_{3}$ and the crystalline aluminosilicate ZSM-5-P were first investigated, exemplifying the influence of acid properties (type of acid sites, acid strength, acid density) and the features of non-microporous (mesoporous) and microporous materials, respectively (Fig. S2 and Table S3). Comparison of the chromatograms of 4-EP conversion over ZSM-5-P and $\gamma-\mathrm{Al}_{2} \mathrm{O}_{3}$ (Fig. 4) shows more

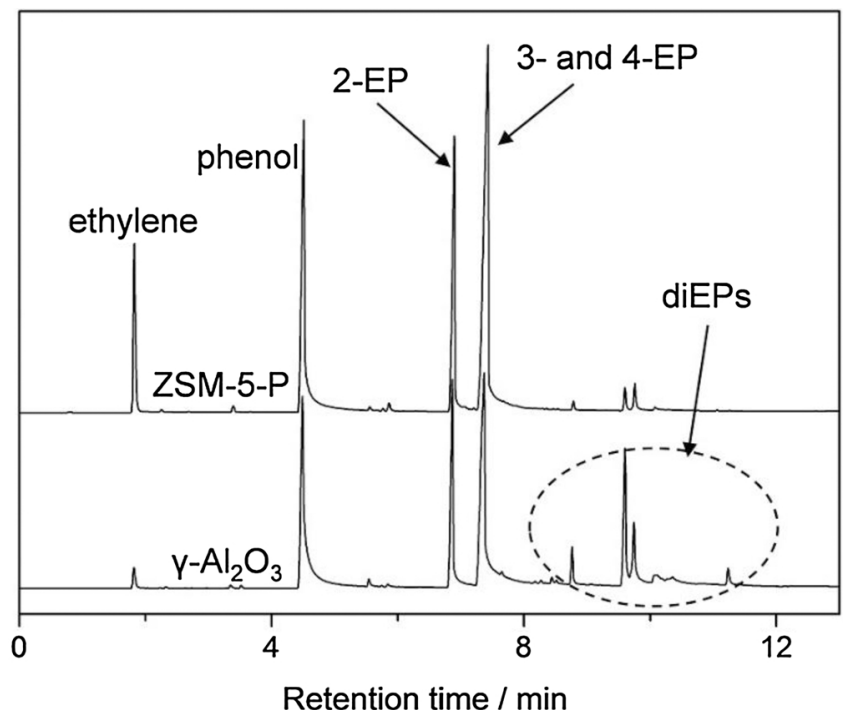

Fig. 4. Gas chromatograms of ZSM-5-P $(638 \mathrm{~K})$ and $\gamma-\mathrm{Al}_{2} \mathrm{O}_{3}(593 \mathrm{~K})$ in the dealkylation of 4-EP at similar conversion of 3- and 4-EP (ca. 65\%). WHSV $=0.027 \mathrm{~mol}$ ( $\mathrm{g}$ cat $)^{-1} \mathrm{~h}^{-1}$, water $/ 4-\mathrm{EP}=6$. The small signals between the ethylene and phenol peak are related to (alkylated) benzenes, whereas the small signals between the phenol and 2-EP peak are related to cresols.

heavy compounds like diEPs in the product stream of $\gamma-\mathrm{Al}_{2} \mathrm{O}_{3}$, suggesting the important role of microporosity and/or acid properties on product distribution. In order to understand their role better and to proof that especially the porosity is an important factor, the product distribution was further investigated as a function of temperature, because the temperature influences not only the conversion rate, but also the thermodynamics of the 4-EP conversion.

Fig. 5 presents the product evolution of 4-EP conversion over various zeolites and $\gamma-\mathrm{Al}_{2} \mathrm{O}_{3}$ in the $473-743 \mathrm{~K}$ temperature range. The dominant observation of 2-EP in Fig. 5a indicates favourable isomerisation of 4-EP at low temperature over ZSM-5-P in agreement with the thermodynamics. Notably, only traces of 4-EP disproportionation and transalkylation products (diEPs) are observed, despite their favourable formation in this temperature region. The dealkylation rate significantly increases with temperature (from $598 \mathrm{~K}$ to $723 \mathrm{~K}$ ), being dominant above $640 \mathrm{~K}$. The yield of cresols and other products like (ethyl) benzene was very low over the entire temperature range in case of ZSM-5-P (Table S5).

Disproportionation, transalkylation and isomerisation are predominant in the catalytic results of $\gamma-\mathrm{Al}_{2} \mathrm{O}_{3}$ in the low temperature range (Fig. 5b). Dealkylation is again the primary reaction in the high 

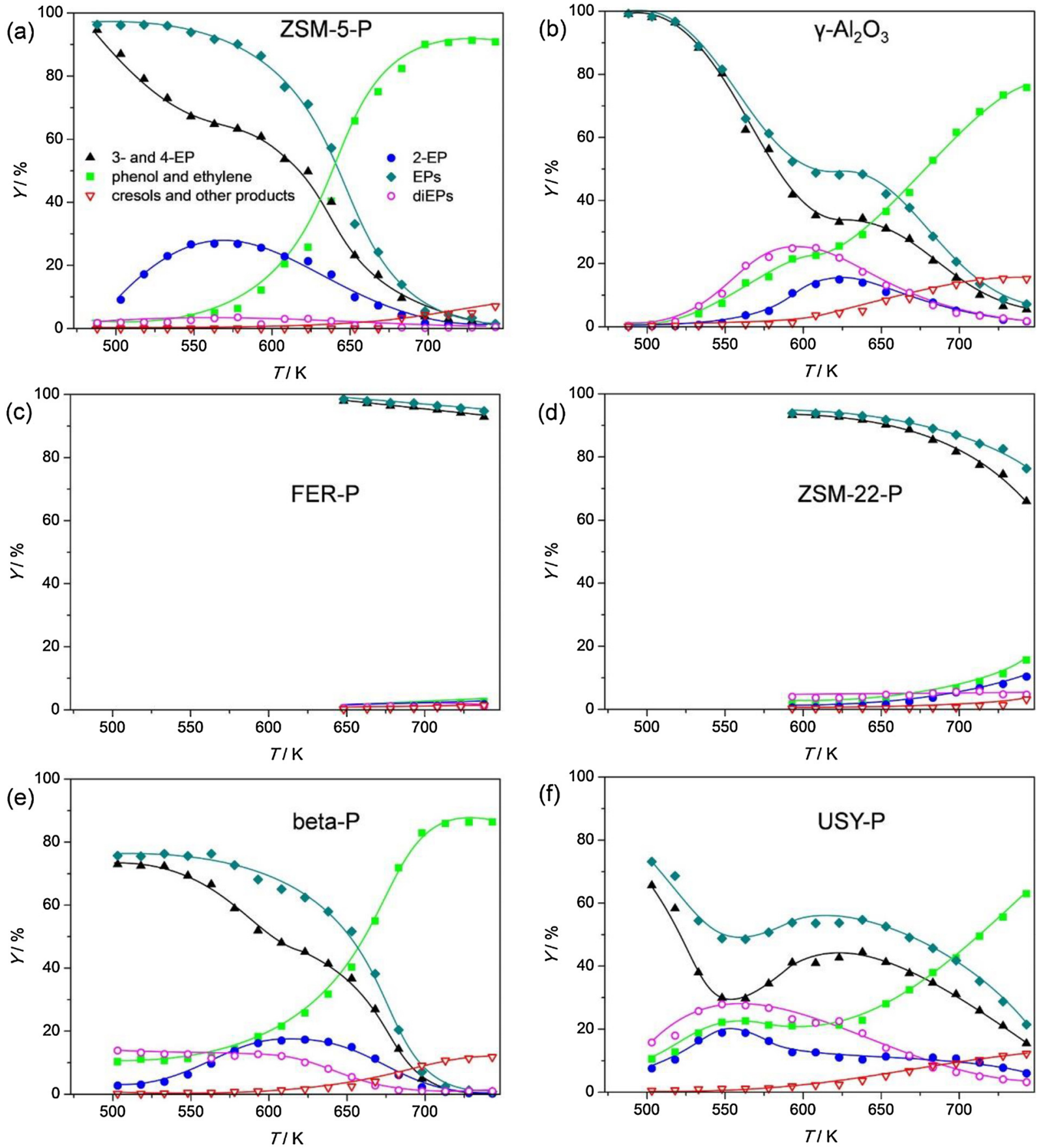

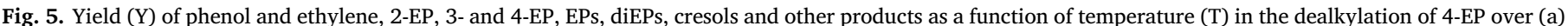

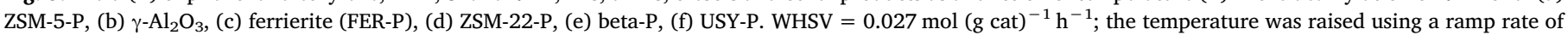
$1 \mathrm{~K} \mathrm{~min}^{-1}$; water/4-EP $=6$. The legend in (a) applies to the entire figure.

temperature range, but the dealkylation selectivity of $\gamma-\mathrm{Al}_{2} \mathrm{O}_{3}$ is substantially lower than that of ZSM-5-P at high temperature due to formation of cresols and other side products (Table S5). The observed results of $\gamma-\mathrm{Al}_{2} \mathrm{O}_{3}$ are fully consistent with the thermodynamics, in that isomerisation, disproportionation and transalkylation are the main reactions at low temperature, whereas dealkylation is favourable at high temperature. Notably, isomerisation occurs at somewhat higher temperature than disproportionation and transalkylation.

To find more evidence in the pore structure effect, more zeolites with distinct topologies ferrierite (FER), ZSM-22 (TON), beta (BEA), and USY (FAU) were tested under similar reaction circumstances. The results are summarized in Fig. 5c-f.

Zeolites with similar Si to $\mathrm{Al}$ ratio ( $c a .40)$ were thus selected as to minimize the impact due to differences in the acid site density or strength. Table S3 shows that the main acid site types in ZSM-22-P, ZSM-5-P, USY-P are Brønsted acids. ZSM-22-P and ZSM-5-P have similar acid density and acid strength (Fig. S3 and Table S3). USY-P gives somewhat lower acid density compared to ZSM-22-P and ZSM-5-P. Beta-P contains the highest Lewis acidity, while the acid strength and acidity of FER-P is lower than that of the other zeolites in agreement with other reports [56].

The zeolites clearly show different catalytic performances in comparable reaction circumstances. For FER-P and ZSM-22-P, conversion of 4-EP only occurs at high temperature (Fig. $5 \mathrm{c}$ and d). The different results of ZSM-22-P and ZSM-5-P indicate that the micropores of zeolites play an important role, as they have similar acidity and acid 
strength. Zeolite beta-P presents a higher conversion rate at low temperature $(T<573 \mathrm{~K})$ than the medium micropore zeolite ZSM-5-P, but it produces mainly diEPs next to EP isomers (Fig. 5e), alike $\gamma-\mathrm{Al}_{2} \mathrm{O}_{3}$ (Fig. 5b), which thus may be assumed due to the presence of nonconfined acid sites. Dealkylation is dominant above $673 \mathrm{~K}$ over beta-P. The phenol selectivity is lower compared to that of ZSM-5-P due to formation of cresols and other side products (Table S5). The isomerisation reaction with beta-P is less pronounced compared to that with ZSM-5-P. The higher conversion rate of beta-P and lower selectivity might be caused by the high (non-confined) Lewis acidity or acid density, but it can also be attributed to the larger micropore size, allowing the occurrence of bimolecular reactions like EP disproportionation.

To distinguish between pore size and (Lewis) acid density, USY-P with its lower acid density and less Lewis acid sites, but larger micropores and similar acid strength (compared to beta-P) was further investigated. USY-P shows a significantly different catalytic profile compared to the other zeolites (Fig. 5f). At low temperature, disproportionation and transalkylation products (diEPs) are predominant, whereas a substantial amount of cresols and other side products are present at high temperature (Table S5), albeit somewhat less than with $\gamma-\mathrm{Al}_{2} \mathrm{O}_{3}$. The evolution of 4-EP over USY-P with temperature is remarkable. After a rapid decrease in the $498 \mathrm{~K}$ to $548 \mathrm{~K}$ temperature range, the yield suddenly shows an increase in the $548 \mathrm{~K}$ to $648 \mathrm{~K}$ temperature range. This increase is accompanied with a decrease of diEPs. This observation agrees with the thermodynamics, shifting from disproportionation and transalkylation to dealkylation with temperature. Dealkylation of diEPs to EPs occurs at intermediate temperature, while full dealkylation to phenol and ethylene proceeds at high temperature. Dealkylation of diEPs leads to 3- and 4-EP. This is consistent with earlier findings showing preferential cracking of 2,4diEP at the ortho position to the hydroxyl group [38]. This might be explained by a specific adsorption geometry of phenols leading to the ortho position close to the active sites, but we have not studied this further here. Isomerisation of diEPs also occurs (not shown). Though $\gamma$ $\mathrm{Al}_{2} \mathrm{O}_{3}$ and USY-P own different type of acid sites and acidity, the reaction outcome is very comparable. Hence, both (spatially unconstraint) Brønsted and Lewis acid sites catalyse the same reactions.

Despite the difference in the extent to which the individual reactions (e.g., dealkylation, isomerisation, and disproportionation) occur, all zeolite catalysts show similar apparent activation energy values (vide supra), suggesting all reactions proceed through the same surface intermediate in the rate determining step. The catalytic results of different aluminosilicates indicate that, although the acid properties influence the catalytic performance (like activity) of aluminosilicates, the selectivity strongly depends on the pore structure, while it is less influenced by the acid properties.

ZSM-5-P is thus the most selective dealkylation catalyst among the evaluated zeolite topologies in the medium to high temperature range (when tested at the constant WHSV; the influence of contact time on selectivity will be discussed later). This conclusion is further supported by the data in Fig. 6, in which the conversion of EPs (varied based on temperature) is plotted against the phenol and ethylene selectivity. Already at a conversion of $40 \%$, the dealkylation selectivity of ZSM- 5 is very high (ca. 95\%), and it remains high up to almost full conversion. The large micropore zeolites (beta and USY) allow the more favourable disproportionation at intermediate conversion, while at high conversion dealkylation is accompanied by side reactions forming predominately cresols, besides a few others (Fig. S9).

The maximum yield of diEPs is strongly influenced by the micropore size of zeolites (Fig. S10), increasing from 4\% for ZSM-5-P to almost $30 \%$ for USY-P. Formation of diEPs is thus strongly inhibited by the micropore structure of ZSM-5-P. Compared to ZSM-5-P, the phenol yield of FER-P and ZSM-22-P is very low, as explained above, due to the restricted accessibility of their micropores. Since EP can enter the micropores of ZSM-5-P, its high phenol yield is related to the inability of

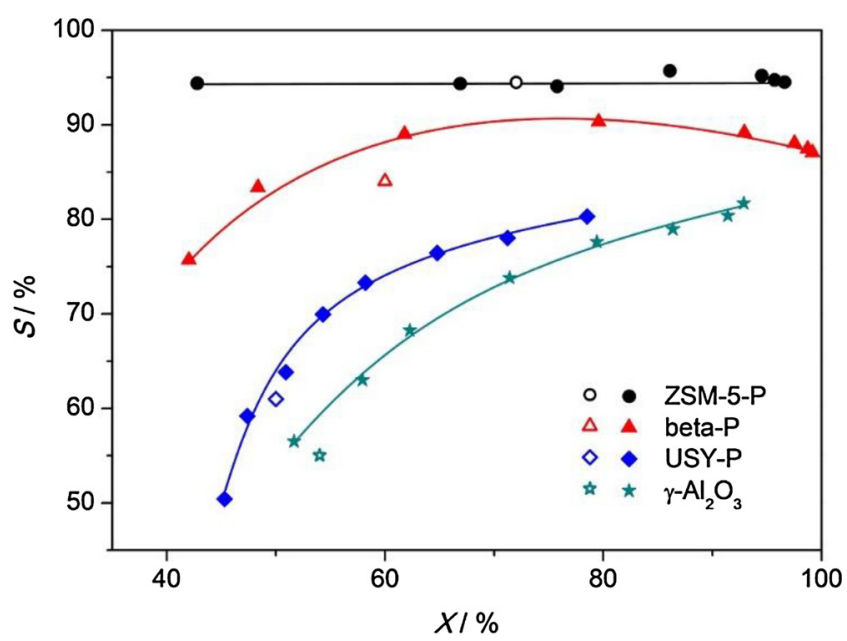

Fig. 6. Selectivity (S) to phenol and ethylene, as a function of conversion $(X)$ of EPs in the transformation of 4-EP over different aluminosilicates. The conversion was changed by increasing the temperature from $473 \mathrm{~K}$ to $743 \mathrm{~K}$ with a ramping rate $1 \mathrm{~K} \mathrm{~min}^{-1}$, WHSV $=0.027 \mathrm{~mol}(\mathrm{~g} \mathrm{cat})^{-1} \mathrm{~h}^{-1}$, water $/ 4-\mathrm{EP}=6$. Hollow symbols are the data from single temperature catalytic test.

EP to disproportionate and transalkylate in the space restricted pores. Such pore constraint effect may be categorized as an example of transition state shape selectivity. Steric restriction of the more thermodynamically favourable reactions not only explains the high selectivity; in part, it also clarifies the lower activity of ZSM-5-P (compared to beta and USY, Fig. 2b).

In addition to selectivity and yield, the stability of ZSM-5-P, beta-P, USY-P, and $\gamma-\mathrm{Al}_{2} \mathrm{O}_{3}$ was investigated at similar conversion rate. Though the presence of water improves the catalytic stability of the zeolite reactions, Beta-P, USY-P, and $\gamma-\mathrm{Al}_{2} \mathrm{O}_{3}$ show moderate deactivation, viz. 7-14\% loss of activity between 2 and $4 \mathrm{~h}$ on stream (Figs. 7a and S11). However, ZSM-5-P is more stable, showing an insignificant 3\% deactivation over the same time period. Photographs of spent catalysts show that the colours of USY-P and ZSM-5-P are black and grey (Fig. S12), respectively, indicating more coking on USY-P and less on ZSM-5-P. The carbon contents of spent ZSM-5-P and USY-P are 3.7\% and 4.5\%, respectively. Coke types are different. USY-P forms more heavy coke, with higher oxidation resistance in the TGA experiment (Fig. 7b: oxidation
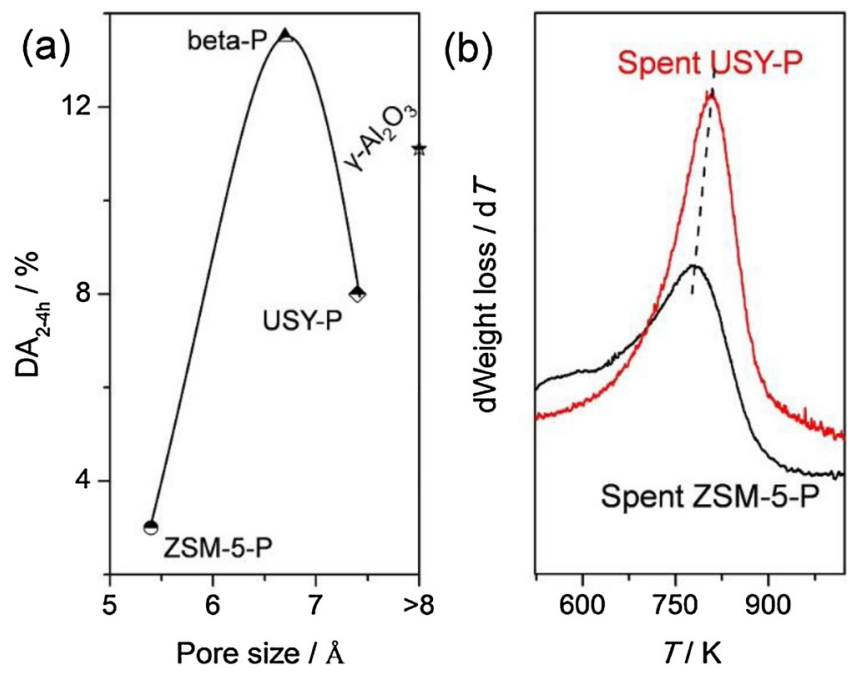

Fig. 7. (a) Deactivation $\left(\mathrm{DA}_{2-4 \mathrm{~h}}\right)$ measured after two hours, as a function of pore size of zeolites. They are calculated from the stability test (Fig. S4), DA 2 $4 \mathrm{~h}=\left(X_{2}-X_{4}\right) / X_{2} \times 100 \%$, where $X_{2}$ is the conversion rate of EPs at time $2 \mathrm{~h}$ and $X_{4}$ is the conversion rate of EPs at time $4 \mathrm{~h}$. (b) Derivation of TGA of the spent ZSM-5-P and USY-P (these samples are from Fig. S4). 
(a)

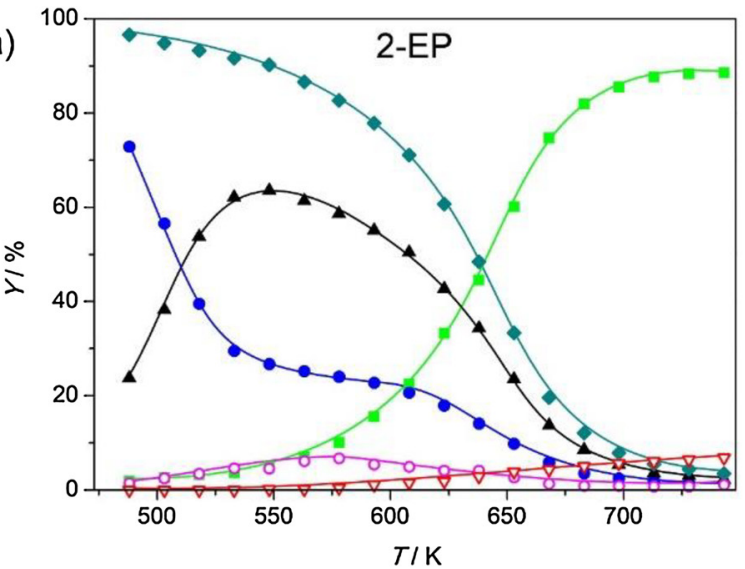

(c)

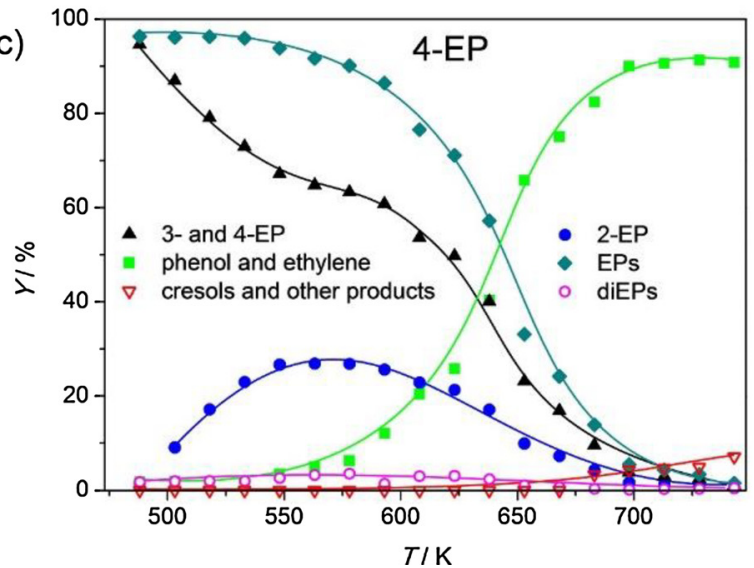

(b)
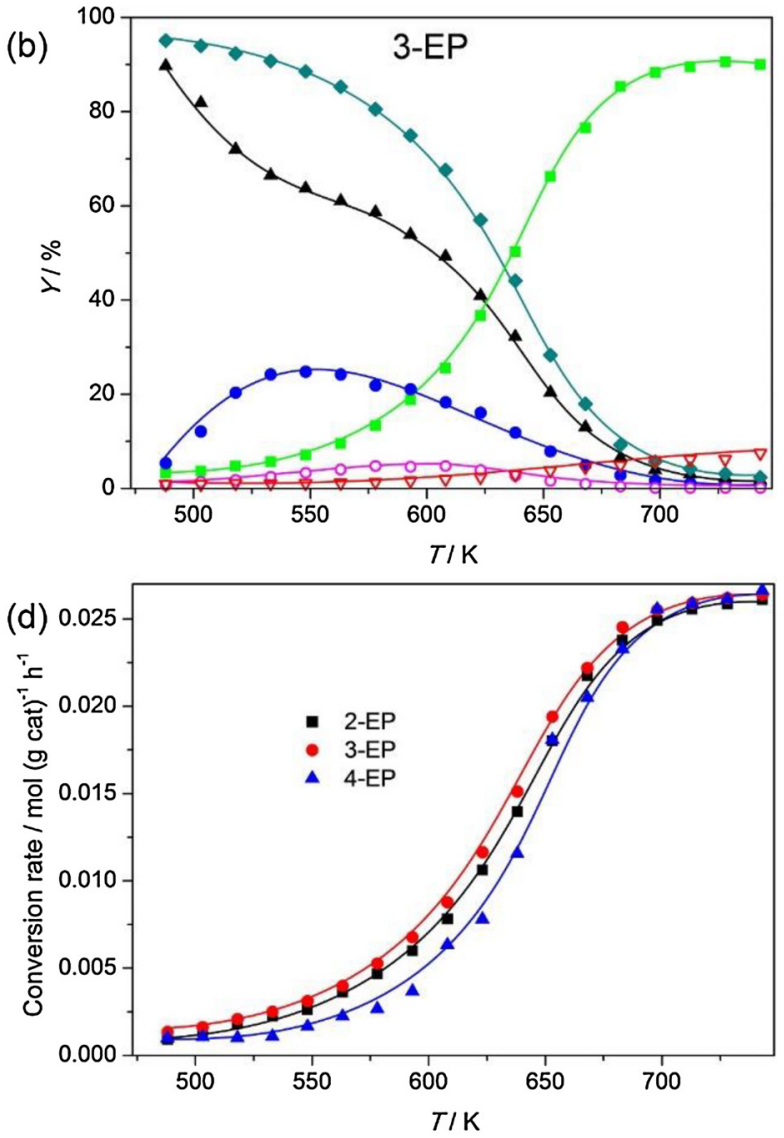

Fig. 8. Catalytic dealkylation of (a) 2-EP, (b) 3-EP (containing $20 \mathrm{wt} . \%$ 4-EP), and (c) 4-EP over ZSM-5-P. (d) Conversion rate of EPs as a function of temperature (T).

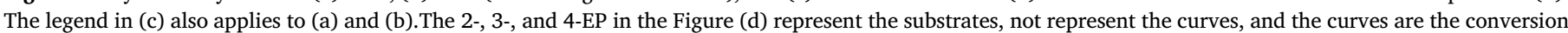
rate of EPs (including 2-, 3-, 4-EP). The temperature was raised using a ramp rate of $1 \mathrm{~K} \mathrm{~min}^{-1} ; \mathrm{WHSV}=0.027 \mathrm{~mol}(\mathrm{~g} \mathrm{cat}){ }^{-1} \mathrm{~h}^{-1}$; water/EP $=6$.

temperature of 808 vs. $778 \mathrm{~K}$ for USY-P and ZSM-5-P, respectively). This illustrates that the medium sized micropores of ZSM-5-P prevent coke fouling superior to the large micropores of USY-P, in agreement with earlier reported results in cresol conversion over ZSM-5 and USY, and in other related reactions [57-59]. In conclusion, ZSM-5-P is the most effective (selective and stable) catalyst to dealkylate 4-EP into phenol and ethylene. Accordingly, the remainder of this work focuses on ZSM-5 zeolites.

\subsection{Isomeric EPs as substrate}

Although lignin-derived EPs are rich in 4-EP, 2- and 3-EP are abundant in coal tar [3]. Besides, 4-EP rapidly converts to 2- and 3-EP (either directly via isomerization or indirectly via dealkylation of diEPs) over acid sites. Hence, study of the catalytic dealkylation of these isomers over ZSM-5-P is also relevant from not only a practical, but also a mechanistic fundamental point of view. The observed products in the transformation of 2-EP, 3-EP and 4-EP illustrate that EP isomers undergo similar reactions, mainly isomerisation at low temperature and dealkylation at high temperature (Figs. 8 and S13). The conversion of 2EP shows a higher EP isomerisation yield than that of 3- and 4-EP (Fig. 8a). This is in line with the thermodynamic equilibrium between the different isomers [60], as calculated in Fig. 1b. The ratio of 3- and 4EP to 2-EP in the conversion of 2-EP and 4-EP indeed converges to a value of 2.5 after the maximum isomer EP yield attained. This value equals the thermodynamic prediction (Fig. 9). This isomer equilibrium is obtained already at $550 \mathrm{~K}$ at the applied WHSV of $0.027 \mathrm{~mol}$ ( $\mathrm{g}$ cat $)^{-1} \mathrm{~h}^{-1}$. This result confirms the fast thermodynamic equilibrium between the isomers in the applied conditions, and appeals against pore diffusion limitation in agreement with above results.

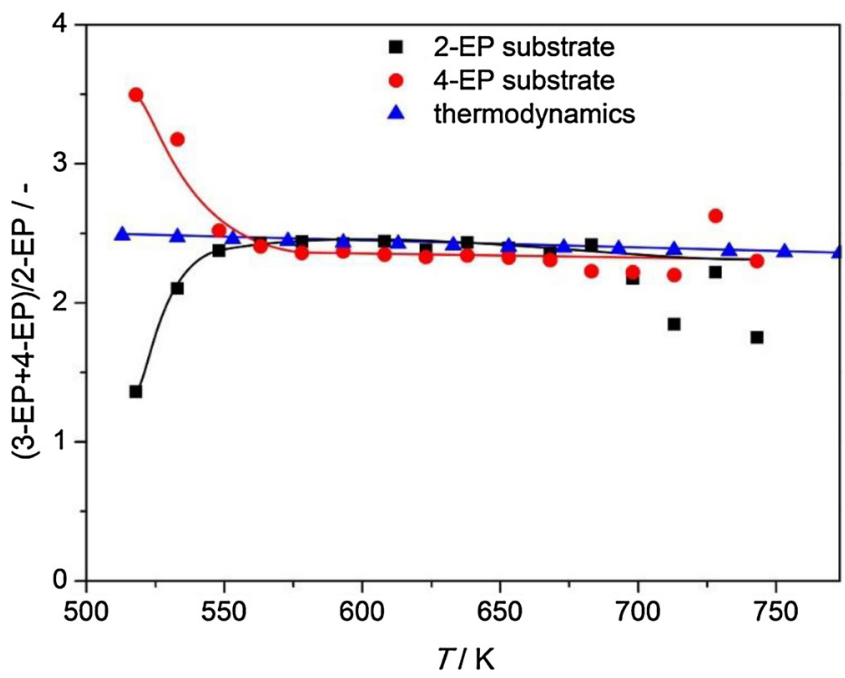

Fig. 9. The (3-EP + 4-EP)/2-EP ratio as a function of temperature $(T)$. Square symbols: experimental values from dealkylation of 2-EP over ZSM-5-P; Circle symbols: experimental values from dealkylation of 4-EP over ZSM-5-P; Up triangle symbols: thermodynamic calculated results (from Fig. 1b).

As ZSM-5-P ultimately leads to the dealkylation of all isomeric EPs, the conversion rate of EPs (including 2-, 3-, and 4-EP) is plotted as a function of temperature in Fig. 8d. The plot displays only a small difference among the isomeric EPs, indicating ZSM-5-P is able to fully dealkylate the mixture of isomeric EPs. Besides, the structure of EPs has practically no influence on the selectivity to phenol and ethylene (Fig. 8 

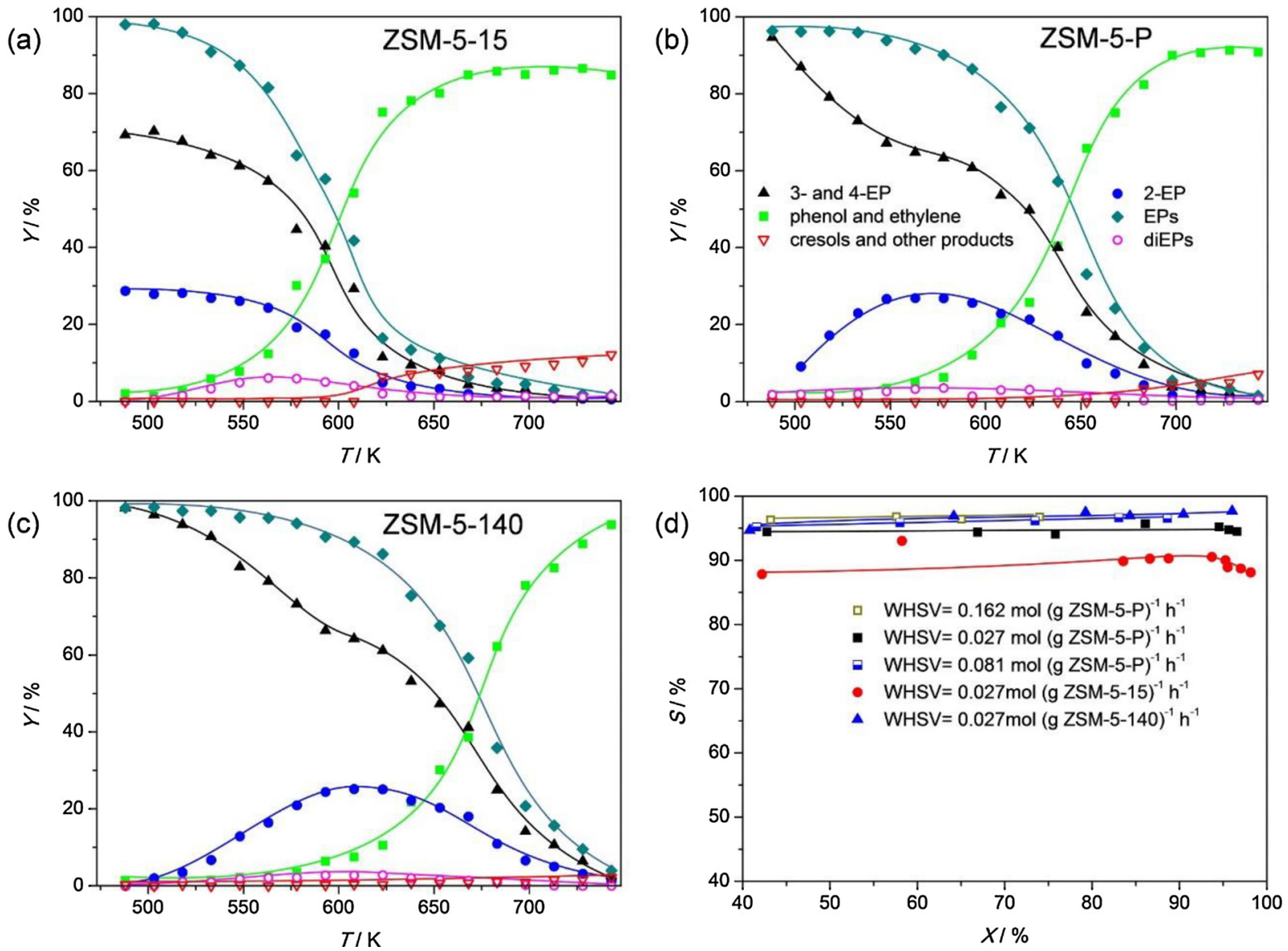

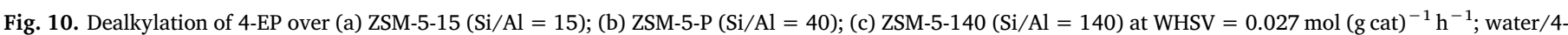

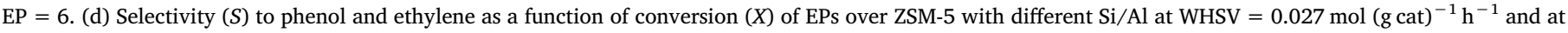
different WHSV over ZSM-5-P; The legend in (b) also applies to (a) and (c).

and Table S5). These results show the great versatility of ZSM-5 to valorise also coal-derived EPs, since mixtures of 2-, 3-, and 4-EP can be converted simultaneously avoiding the necessity of energy intensive separation processes.

\subsection{Effect of the contact time of EP with the acidic sites}

Variation of the contact time of substrate to the active sites is an important tool to determine the reaction network, being able to distinguish between primary and secondary reactions. The contact time can be varied either by changing the active site density or by varying WHSV. ZSM-5 samples with different Si to Al ratio (from 15 to 140; ZSM-5-15, ZSM-5-P and ZSM-5-140), hence different acid site density (Table S3), were investigated first. The Arrhenius plots are presented in Fig. 2b, while the product distribution in function of temperature is plotted in Fig. 10a-c. Notably, all ZSM-5 samples show similar apparent activation energies, viz. $60 \mathrm{~kJ} \mathrm{~mol}^{-1}$, while the turnover frequencies (TOFs) of ZSM-5 are only slightly different (Fig. 2b). The minor difference might be due to polarity effects (as a result of the $\mathrm{Si}$ to $\mathrm{Al}$ ratio difference, and therefore different 4-EP loadings in the zeolite occur) or differences in acid strength (Fig. S3), but any explanation needs further quantitative support.

With decreasing $\mathrm{Si}$ to $\mathrm{Al}$ ratio, the temperature to get the same yield of phenol and ethylene is reduced and this effect is thus largely due to the increasing number of active acid sites (Fig. 10 and Table S3). Besides, lower temperature is needed to reach the maximum yield of 2-EP over the lower $\mathrm{Si}$ to $\mathrm{Al}$ ratio. The maximum isomer yield (monitored by 2-EP formation) is obtained at similar conversion (ca. 35\%), and therefore independent on the acid sites density (Fig. S14). Since the amount of Lewis acid sites that are located at the external surface of ZSM-5 (which is unconstrained) increases with decreasing Si to $\mathrm{Al}$ ratio (Table S3) [61,62], a higher yield of diPPs from disproportionation and transalkylation is indeed obtained. Fig. 10d shows that the selectivity to phenol and ethylene is slightly decreased with increasing acid site density (decreasing $\mathrm{Si}$ to $\mathrm{Al}$ ratio), for the same reason.

Variation of WHSV (by reducing catalyst weight; see experimental section) was also studied for 4-EP conversion over ZSM-5-P. The data are presented in the Arrhenius plot of Fig. 2b. Notably, the variation of WHSV has no significant impact on the apparent activation energy and on the pre-exponential factor. As it should be, a higher contact time (more catalyst for the same feed flow) shows a higher conversion rate in the same reactions conditions (Figs. S15 and S16). Fig. 10d shows that the selectivity to phenol and ethylene increases slightly with increasing WHSV (decreasing contact time), suggesting some impact of secondary reactions on the product selectivity. Therefore, highest selectivity and conversion rate can be achieved by balancing the contact time and reaction temperature. In this work, a phenol and ethylene productivity of $8.34 \mathrm{~g}$ (g cat) ${ }^{-1} \mathrm{~h}^{-1}$ (with $96 \%$ phenol and ethylene selectivity) is obtained with a ZSM-5-P at $743 \mathrm{~K}$ using a WHSV of $0.081 \mathrm{~mol}$ (g cat) $)^{-1} \mathrm{~h}^{-1}$.

In addition to the influence of acid site density on the selectivity to phenol and ethylene, its influence on the catalytic stability was evaluated at similar productivity (and also similar conversion). The results show that all ZSM- 5 zeolites reach a quite stable situation within a $4 \mathrm{~h}$ test, but the selectivity is somewhat lower with increasing the acid sites number (Fig. 11a). The ZSM-5 catalyst deactivation on stream shows a classic decay behaviour that can be fitted by empirical formula (Fig. S17). Usually the initial exponential decay is caused by coking of the 

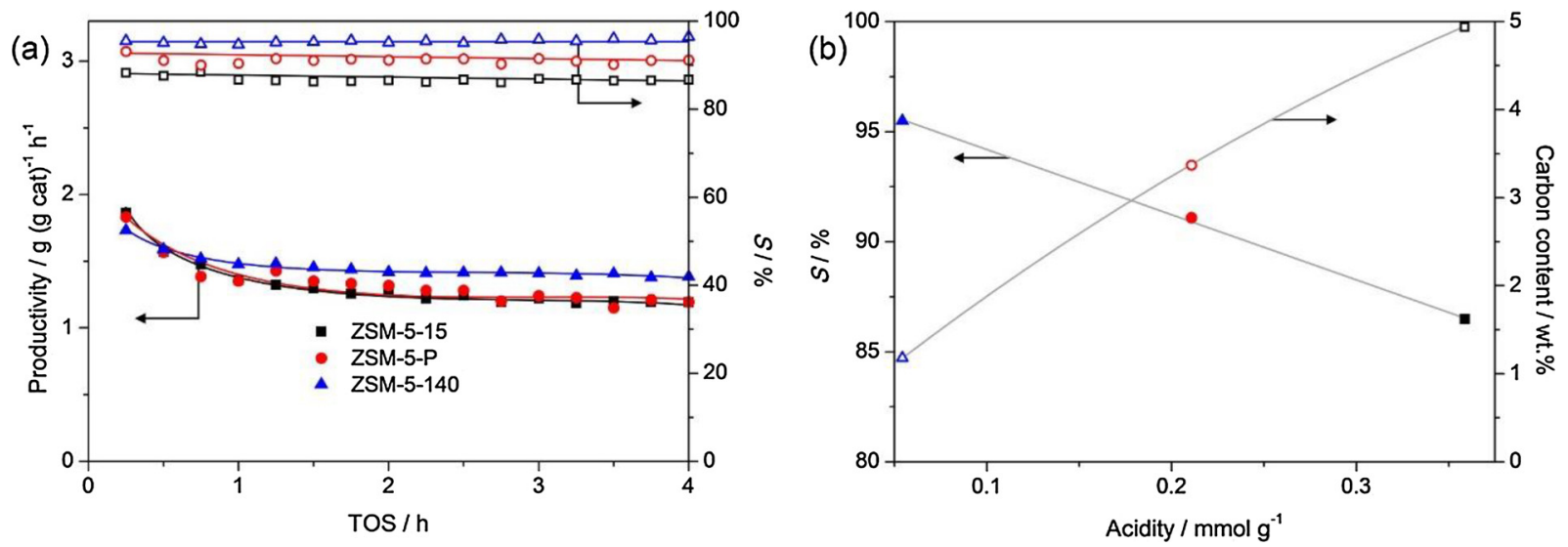

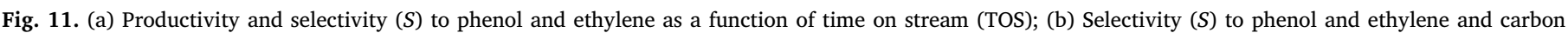

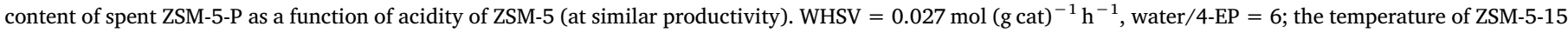
is $593 \mathrm{~K}$, ZSM-5-P is $623 \mathrm{~K}$, and ZSM-5-140 is $668 \mathrm{~K}$.

external surface acid sites, while the minor linear decay over time is due to pore narrowing by the slowly growing cokes [63]. Obviously, the higher density of surface active sites with decreasing $\mathrm{Si} / \mathrm{Al}$ ratio aligns with the larger deactivation of ZSM-5-15 compared to ZSM-5-140. Accordingly, the colours of spent ZSM-5 gradually darken with increasing number of acid sites (Fig. S18). As is generally observed in the transformation of hydrocarbons [64], the catalyst with the most sites is accompanied by the highest coke formation (Fig. 11b). This observation implies that ZSM-5 featuring the lowest acid site density, here ZSM-5140 , is the most stable catalyst.

\subsection{Reaction pathway}

The reaction pathways involved in 4-EP conversion are isomerization, dealkylation, disproportionation, and transalkylation. The different reactions are shown in Scheme 1 and Fig. S19. The conversion reaction of 4-EP over the zeolites proceeds by protonation of the 4-EP molecule over the Brønsted acid sites (Scheme 1a), and by hydride ion abstraction of 4-EP over the Lewis acid sites (Fig. S19). A 1,2-ethyl shift reaction is the origin of the isomerisation forming 3-EP, and then 2-EP (intramolecular isomerisation), which is similar to the isomerisation mechanism of cresol and xylene [65,66]. Such shift reactions are possible in ZSM-5 and wide-pore zeolites. The activated intermediate may also react with another 4-EP molecule via $\mathrm{S}_{\mathrm{N} 2}$ mechanism, forming the diphenylethane intermediate that disproportionates into phenol and diEPs (disproportionation). Besides, the formation of diEPs may also follow a $\mathrm{S}_{\mathrm{N} 1}$ mechanism like in xylene disproportionation. In this case, 4-EP first dealkylates into an ethyl ion and a phenol molecule. The former then alkylates another 4-EP molecule to form diEPs. Such 4-EP disproportionation can be restricted in the pores of ZSM-5, while there is sufficient room to accommodate the diphenylethane intermediate in the wide-pores of USY and beta. Transalkylation is the transfer of the ethyl group from 4-EP to another different molecule like 2-EP to form diEPs and phenol, which is also a bimolecular process. While substantially limited in the zeolite pore of ZSM-5, it occurs in the pores of beta and USY.

Dealkylation occurs through a monomolecular reaction, which is only effective at high temperature for both kinetic and thermodynamics reasons. The mechanistic pathway implies a direct scission of the $\mathrm{C}_{\mathrm{Ar}^{-}}$ $\mathrm{C}_{2} \mathrm{H}_{5}$ bond, generating ethylene as the product. This is not similar to the more classical behaviour of hydrodealkylation of alkylbenzene or thermal cracking of EP.[67] In that case, the alkyl group undergoes a successive cleavage of the terminal $\mathrm{C}-\mathrm{C}$ bond, yielding (the less valuable) methane as the main product of alkyl group. [67]

The catalytic results of ZSM-5 indicate that isomerisation reaches equilibrium rapidly; the ratio of isomers is independent of the temperature after a certain conversion (ca. 30\%). Since there is no isomer production deviating from the thermodynamic equilibrium, the effect of the different diffusivities of the isomers on the kinetics is limited on the tested ZSM-5-P. This effect is often referred to as 'disguised kinetics', which is also observed in xylene and cresol isomerisation $[66,68]$. Only after a certain minimum crystal size, which is much larger than the ones used here, the off-thermodynamic product distribution due to diffusivity differences is usually noticed. Besides the isomerisation, disproportionation and transalkylation contributions to EP conversion are substantial at low temperature over the large micropore zeolites (like beta-P and USY-P) and $\gamma-\mathrm{Al}_{2} \mathrm{O}_{3}$, but not over ZSMP. Spatial constraints of the medium pore zeolite ZSM-5 strongly inhibits the bimolecular reactions through transition state shape selectivity in the micropores. For FER-P and ZSM-22-P zeolites, all reactions are severely inhibited due to the inability of the reactant molecule to enter the zeolite crystals through the micropores.

At high temperature monomolecular dealkylation is the main pathway over ZSM-5 and large micropore zeolites. The diEPs and isomeric EPs also undergo dealkylation in these conditions. The side reactions (e.g., formations of cresols and other products like ethylbenzene, benzene), which underwent C-C and C-O cracking, are favourable at the high temperature (data not shown), and are more pronounced for beta and USY. This difference between ZSM-5 and USY is attributed to not only the thermodynamics but also the steric effect. The side chain CC cracking to form cresols not only follows a monomolecular pathway, but usually occurs via bimolecular reactions, that further leads to cracking (Scheme 1b) [69]. The monomolecular reaction can involve thermal cracking, as demonstrated in the pyrolysis of EPs, and catalytic cracking of the $\mathrm{C}-\mathrm{C}$ bond of the ethyl group with hydrogen transfer [32]. For the bimolecular reactions, the diphenylethane intermediates are different from those of disproportionation and transalkylation to diEPs, as shown in Scheme 1b. The intermediates are formed via terminal methyl transfer from one 4-EP molecule to another molecules like 4-EP, produced isomers (not shown), and phenol. Afterwards, these intermediates can undergo C-C cracking to form (ethyl) cresols (Scheme 1b). The cresols may further undergo isomerization over the acidic catalysts (not shown). For steric reasons, zeolites with relatively narrow pores, such as ZSM-5, restrict these space-demanding bimolecular reactions. This is consistent with the low yield of cresols for ZSM-5, compared to that of large micropore zeolites such as USY or beta. Similar results have been reported for alkylbenzene like cumene and ethylbenzene cracking over different acid zeolites [70]. All together, the selectivity phenomenon illustrates that the medium micropore zeolite ZSM-5 favours monomolecular reactions (isomerisation and dealkylation), and the bimolecular reactions (like disproportionation and transalkylation) is severely inhibited, whereas large micropores 

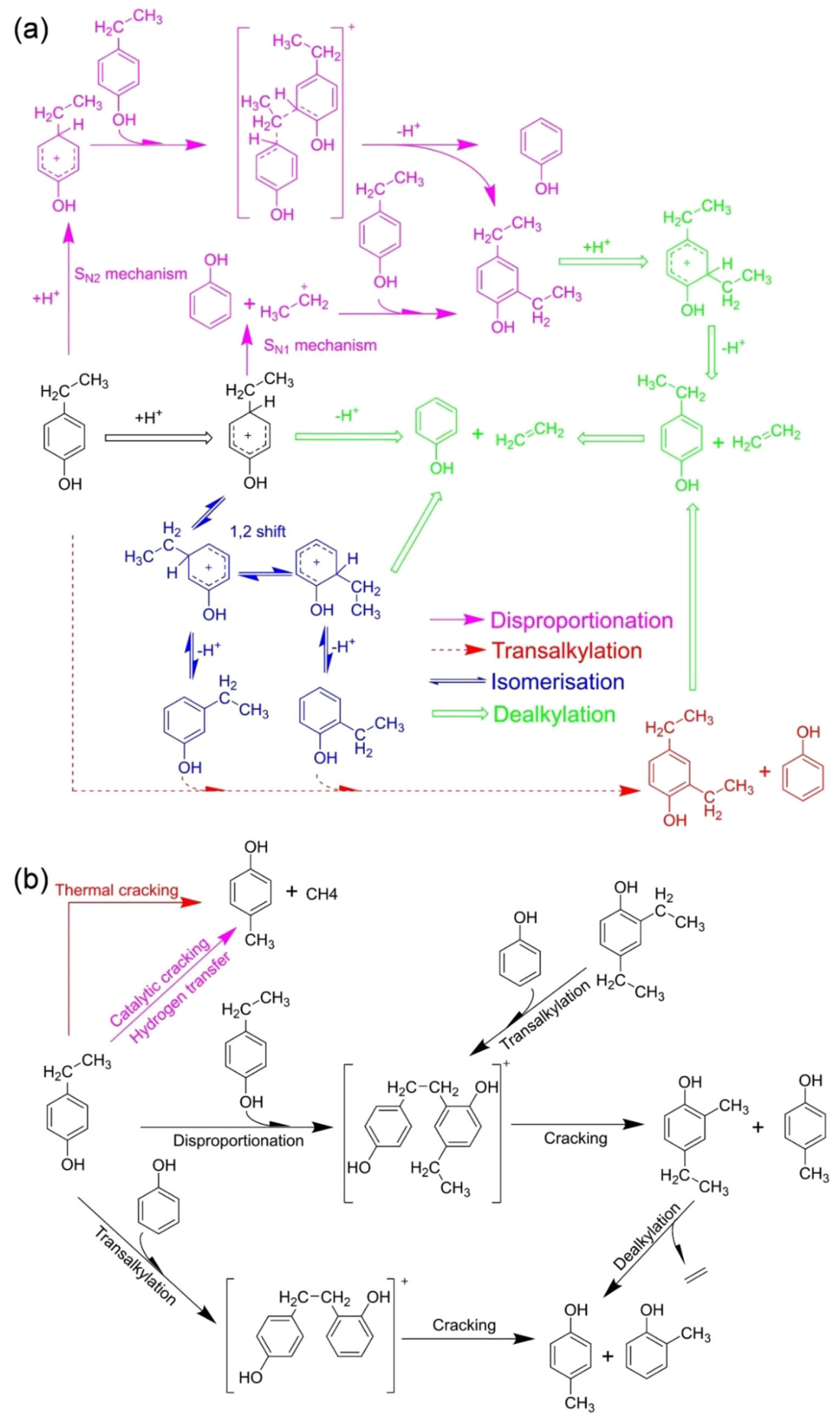

Scheme 1. (a) Proposed main reaction pathway of 4-EP conversion over Brønsted acid sites of aluminosilicates; (b) Proposed reaction pathway of cresols formation. 
like USY enable both monomolecular and bimolecular reaction pathways. The role of micropores of zeolites for 4-EP conversion therefore shows strong resemblance to the conversion of ethylbenzene over medium or large pore zeolites [51,52].

\section{Conclusion}

In the conversion of ethylphenols (EP) in presence of steam, Brønsted and Lewis acid sites are both identified as active sites. Different reactions such as isomerisation, disproportionation, dealkylation, C-C and C-O cracking are involved in the reaction network. Isomerisation, disproportionation and transalkylation dominate at low temperature (e.g., $473 \mathrm{~K}<\mathrm{T}<623 \mathrm{~K}$ ), whereas at high temperatures (e.g., $\mathrm{T}>623 \mathrm{~K}$ ) monomolecular dealkylation is thermodynamically favoured. The activity of the catalyst is influenced by the acid properties such as strength and type of actives sites, while also the pore size is a dominant factor. Access through the micropores is essential, ZSM-5-P and wide-pore zeolites like beta-P and USY-P therefore offering a high activity, whereas low activity is observed with FER-P and ZSM-22-P. The higher activity of wide-pore zeolites is not due to pore diffusion limitation in the zeolite pores, as evidenced in several different ways, but it finds explanation in a reaction pathway shift. Large pore zeolites allow the faster and thermodynamically more favourable bimolecular reactions, and therefore they show high conversion rates, whereas medium pore zeolite ZSM-5 converts EP through monomolecular reactions. This shift impacts also the reaction selectivity in favour of the medium-pore zeolites. Medium-pore ZSM- 5 is identified as the most selective and catalytically stable catalyst, which is ascribed to the optimal constraints, which EPs experience in its micropores. Herein, it is suggested that a restricted transition state selectivity, a type of shape selectivity that is possible in absence of pore diffusion, strongly inhibits the bimolecular reactions at the origin of disproportionation, transalkylation (at intermediate temperature) and side chain C-C cracking (at high temperature). Optimization of the contact time, through the acid site density and WHSV, further improves the selectivity and productivity to phenol and ethylene. Catalyst deactivation due to cokes formation is less pronounced with the medium-pore zeolite ZSM-5 due to pore restriction. Initial exponential decay fits with surface coking, more exposed for ZSM-5 with higher (surface) site density and unconfined surface Lewis acid sites, followed by a slow linear deactivation slope due to pore narrowing. There is a minor contribution of loss in phenol selectivity due to secondary reactions, therefore preferring low contact time conditions. The excellent catalytic behaviour of acidic zeolites, especially ZSM-5, in the dealkylation of EP showing almost quantitative yields of phenol and ethylene, corresponding to a productivity of $8.34 \mathrm{~g}$ ( $\mathrm{g} \mathrm{cat})^{-1} \mathrm{~h}^{-1}$, illustrates the potential of zeolites to valorise biomass- and coal-derived alkylphenols into base chemicals such as phenol and olefins.

\section{Acknowledgements}

The authors are grateful to $\mathrm{W}$. Vermandel for technical assistance during the catalytic testing and $\mathrm{NH}_{3}$-TPD measurements, and Dr. Roel Locus for pyridine FTIR measurements, Dr. R. Shu for elemental analysis. Y. L. acknowledges funding from China Scholarship Council (CSC) for a doctoral fellowship (201404910467). D. V. thanks funding from the Research Foundation Flanders (FWO) for a post-doctoral fellowship. FISCH-ARBOREF financial support by the Flemish government and Excellence of Science BIOFACT financial support by the Belgian government are acknowledged.

\section{Appendix A. Supplementary data}

Supplementary material related to this article can be found, in the online version, at doi:https://doi.org/10.1016/j.apcatb.2018.04.001.

\section{References}

[1] C. Li, X. Zhao, A. Wang, G.W. Huber, T. Zhang, Chem. Rev, 115 (2015) 11559-11624.

[2] J. Zakzeski, P.C.A. Bruijnincx, A.L. Jongerius, B.M. Weckhuysen, Chem. Rev. 110 (2010) 3552-3599.

[3] H. Fiege, H.W. Voges, T. Hamamoto, S. Umemura, T. Iwata, H. Miki, Y. Fujita, H.J. Buysch, D. Garbe, W. Paulus, Phenol derivatives, Ullmann's Encyclopedia of Industrial Chemistry, John Wiley \& Sons, 2000.

[4] D. Verboekend, Y. Liao, W. Schutyser, B.F. Sels, Green Chem. 18 (2016) 297-306.

[5] C. Xu, R.A. Arancon, J. Labidi, R. Luque, Chem. Soc. Rev. 43 (2014) 7485-7500.

[6] T. Renders, W. Schutyser, S. Van den Bosch, S.F. Koelewijn, T. Vangeel, C.M. Courtin, B.F. Sels, ACS Catal. 6 (2016) 2055-2066.

[7] E.M. Anderson, R. Katahira, M. Reed, M.G. Resch, E.M. Karp, G.T. Beckham, Y. Román-Leshkov, ACS Sustain. Chem. Eng. 4 (2016) 6940-6950.

[8] H. Luo, I.M. Klein, Y. Jiang, H. Zhu, B. Liu, H.I. Kenttämaa, M.M. Abu-Omar, ACS Sustain. Chem. Eng. 4 (2016) 2316-2322.

[9] S. Van den Bosch, W. Schutyser, S.F. Koelewijn, T. Renders, C.M. Courtin, B.F. Sels, Chem. Commun. 51 (2015) 13158-13161.

[10] M.V. Galkin, J.S.M. Samec, ChemSusChem 7 (2014) 2154-2158.

[11] M.V. Galkin, J.S.M. Samec, ChemSusChem 9 (2016) 1544-1558.

[12] N. Yan, C. Zhao, P.J. Dyson, C. Wang, L. Liu, Y. Kou, ChemSusChem 1 (2008) 626-629.

[13] Q. Song, F. Wang, J. Cai, Y.H. Wang, J. Zhang, W.Q. Yu, J. Xu, Energy Environ. Sci. 6 (2013) 994-1007.

[14] S. Van den Bosch, W. Schutyser, R. Vanholme, T. Driessen, S.F. Koelewijn, T. Renders, B. De Meester, W. Huijgen, W. Dehaen, C.M. Courtin, B. Lagrain, W. Boerjan, B.F. Sels, Energy Environ. Sci. 8 (2015) 1748-1763.

[15] P. Azadi, R. Carrasquillo-Flores, Y.J. Pagan-Torres, E.I. Gurbuz, R. Farnood, J.A. Dumesic, Green Chem. 14 (2012) 1573-1576.

[16] T. Parsell, S. Yohe, J. Degenstein, T. Jarrell, I. Klein, E. Gencer, B. Hewetson, M. Hurt, J.I. Kim, H. Choudhari, B. Saha, R. Meilan, N. Mosier, F. Ribeiro, W.N. Delgass, C. Chapple, H.I. Kenttamaa, R. Agrawal, M.M. Abu-Omar, Green Chem. 17 (2015) 1492-1499.

[17] W. Schutyser, S. Van den Bosch, T. Renders, T. De Boe, S.F. Koelewijn, A. Dewaele, T. Ennaert, O. Verkinderen, B. Goderis, C.M. Courtin, B.F. Sels, Green Chem. 17 (2015) 5035-5045.

[18] T. Renders, S. Van den Bosch, S.F. Koelewijn, W. Schutyser, B. Sels, Energy Environ. Sci. 10 (2017) 1551-1557.

[19] E.M. Anderson, M.L. Stone, R. Katahira, M. Reed, G.T. Beckham, Y. Román-Leshkov, Joule 1 (2017) 613-622.

[20] W. Schutyser, T. Renders, S. Van den Bosch, S.F. Koelewijn, G.T. Beckham, B.F. Sels, Chem. Soc. Rev. 47 (2018) 852-908.

[21] Z. Jiang, T. He, J. Li, C. Hu, Green Chem. 16 (2014) 4257-4265.

[22] L. Hu, Y. Luo, B. Cai, J. Li, D. Tong, C. Hu, Green Chem. 16 (2014) 3107-3116.

[23] Q. Song, F. Wang, J. Xu, Chem. Commun. 48 (2012) 7019-7021.

[24] S. Van den Bosch, T. Renders, S. Kennis, S.F. Koelewijn, G. Van den Bossche, T. Vangeel, A. Deneyer, D. Depuydt, C.M. Courtin, J. Thevelein, W. Schutyser, B.F. Sels, Green Chem. 19 (2017) 3313-3326.

[25] T. Renders, S. Van den Bosch, T. Vangeel, T. Ennaert, S.F. Koelewijn, G. Van den Bossche, C.M. Courtin, W. Schutyser, B.F. Sels, ACS Sustain. Chem. Eng. 4 (2016) 6894-6904.

[26] A. Toledano, L. Serrano, A. Pineda, A.A. Romero, R. Luque, J. Labidi, Appl. Catal. B 145 (2014) 43-55.

[27] Y. Liao, A. Dewaele, D. Verboekend, B.F. Sels, Alkylphenols as Bio-Based Solvents: Properties, Manufacture and Applications, Bio-Based Solvents, John Wiley \& Sons, 2017.

[28] S.F. Koelewijn, S. Van den Bosch, T. Renders, W. Schutyser, B. Lagrain, M. Smet, J. Thomas, W. Dehaen, P. Van Puyvelde, H. Witters, B.F. Sels, Green Chem. 19 (2017) 2561-2570.

[29] W. Schutyser, S. Van den Bosch, J. Dijkmans, S. Turner, M. Meledina, G. Van Tendeloo, D.P. Debecker, B.F. Sels, ChemSusChem 8 (2015) 1805-1818.

[30] Y. Romero, F. Richard, S. Brunet, Appl. Catal. B 98 (2010) 213-223.

[31] S.F. Koelewijn, C. Cooreman, T. Renders, C. Andecochea Saiz, S. Van den Bosch, W. Schutyser, W. De Leger, M. Smet, P. Van Puyvelde, H. Witters, B. Van der Bruggen, B.F. Sels, Green Chem. 20 (2018) 1050-1058.

[32] P. Zhou, B.L. Crynes, Ind. Eng. Chem. Proc. Des. Dev. 25 (1986) 898-907.

[33] P. Zhou, B.L. Crynes, Ind. Eng. Chem. Proc. Des. Dev. 25 (1986) 767-771.

[34] G.L. Wells, R. Long, Ind. Eng. Chem. Proc. Des. Dev. 1 (1962) 73-79.

[35] C. Hu, J. Li, W. Jia, M. Liu, Z. Hao, Z. Zhu, Chin. J. Chem. 33 (2015) 247-252.

[36] T. Sato, T. Adschiri, K. Arai, J. Anal. Appl. Pyrolysis 70 (2003) 735-746.

[37] F.P. Daly, J. Catal. 61 (1980) 528-532.

[38] M. Kraus, V. Bažant, Collect. Czech. Chem. Commun. 28 (1963) 1877-1884.

[39] P. Schneider, M. Kraus, V. Bažant, Collect. Czech. Chem. Commun. 26 (1961) 1636-1645.

[40] P.A. Jacobs, M. Dusselier, B.F. Sels, Angew. Chem. Int. Ed. 53 (2014) 8621-8626.

[41] T. Ennaert, J. Van Aelst, J. Dijkmans, R. De Clercq, W. Schutyser, M. Dusselier, D. Verboekend, B.F. Sels, Chem. Soc. Rev. 45 (2016) 584-611.

[42] E. Taarning, C.M. Osmundsen, X. Yang, B. Voss, S.I. Andersen, C.H. Christensen, Energy Environ. Sci. 4 (2011) 793-804.

[43] N. Nuttens, D. Verboekend, A. Deneyer, J. Van Aelst, B.F. Sels, ChemSusChem 8 (2015) 1197-1205.

[44] C.A. Emeis, J. Catal. 141 (1993) 347-354.

[45] R. Zhong, F. Yu, W. Schutyser, Y. Liao, F. de Clippel, L. Peng, B.F. Sels, Appl. Catal. B 206 (2017) 74-88. 
[46] D.E. Mears, Ind. Eng. Chem. Proc. Des. Dev. 10 (1971) 541-547.

[47] P.B. Weisz, C. Prater, Interpretation of measurements in experimental catalysis, Adv. Catal. 6 (1954) 143-196.

[48] P. Strnad, M. Kraus, Collect. Czech. Chem. Commun. 30 (1965) 1136-1143.

[49] H. Karge, J. Ladebeck, Z. Sarbak, K. Hatada, Zeolites 2 (1982) 94-102.

[50] J. Das, Y.S. Bhat, A.B. Halgeri, Ind. Eng. Chem. Res. 32 (1993) 2525-2529.

[51] J. Huang, Y. Jiang, V.R. Marthala, M. Hunger, J. Am. Chem. Soc. 130 (2008) 12642-12644

[52] J. Huang, Y.J. Jiang, V.R.R. Marthala, A. Bressel, J. Frey, M. Hunger, J. Catal. 263 (2009) 277-283.

[53] https://www.molinstincts.com.

[54] J.J. Talley, Catalytic hydrodealkylation of alkylated phenols, US, 4533767 (1985).

[55] G. Bjornson, Hydrodealkylation process and catalyt, US, 4194844 (1980).

[56] M.B. dos Santos, H.M. Andrade, A.J. Mascarenhas, Microporous Mesoporous Mater. 223 (2016) 105-113.

[57] F.E. Imbert, M. Guisnet, S. Gnep, J. Catal. 195 (2000) 279-286.

[58] M. Guisnet, P. Magnoux, Catal. Today 36 (1997) 477-483.
[59] P. Castaño, G. Elordi, M. Olazar, A.T. Aguayo, B. Pawelec, J. Bilbao, Appl. Catal. B 104 (2011) 91-100.

[60] M. Sad, H. Duarte, C. Padró, C. Apesteguía, Appl. Catal. A 486 (2014) 77-84.

[61] T. Armaroli, L.J. Simon, M. Digne, T. Montanari, M. Bevilacqua, V. Valtchev, J. Patarin, G. Busca, Appl. Catal. A 306 (2006) 78-84.

[62] Y.T. Kim, K.-D. Jung, E.D. Park, Microporous Mesoporous Mater. 131 (2010) 28-36.

[63] E. Santacesaria, M. Di Serio, P. Ciambelli, D. Gelosa, S. Carra, Appl. Catal. 64 (1990) $101-117$.

[64] G. Ertl, H. Knözinger, F. Schüth, J. Weitkamp, Handbook of Heterogeneous Catalysis, 2nd edition, John Wiley \& Sons, 2008.

[65] A. Cortes, A. Corma, J. Catal. 51 (1978) 338-344.

[66] F.E. Imbert, N. Gnep, M. Guisnet, J. Catal. 172 (1997) 307-313.

[67] L. Beranek, M. Kraus, Collect. Czech. Chem. Commun. 31 (1966) 566-575.

[68] R. Prins, A. Wang, X. Li, Introduction to Heterogeneous Catalysis, World Scientific Publishing (UK) Ltd, 2016.

[69] A. Corma, P. Miguel, A. Orchilles, G. Koermer, J. Catal. 145 (1994) 181-186.

[70] J.M. Serra, E. Guillon, A. Corma, J. Catal. 227 (2004) 459-469. 